\title{
COMPARISON OF FASTNESS OF THE CONVERGENCE AMONG KRASNOSELSKIJ, MANN, AND ISHIKAWA ITERATIONS IN ARBITRARY REAL BANACH SPACES
}

\author{
G. V. R. BABU AND K. N. V. V. VARA PRASAD \\ Received 25 April 2006; Accepted 4 September 2006
}

Let $E$ be an arbitrary real Banach space and $K$ a nonempty, closed, convex (not necessarily bounded) subset of $E$. If $T$ is a member of the class of Lipschitz, strongly pseudocontractive maps with Lipschitz constant $L \geq 1$, then it is shown that to each Mann iteration there is a Krasnosleskij iteration which converges faster than the Mann iteration. It is also shown that the Mann iteration converges faster than the Ishikawa iteration to the fixed point of $T$.

Copyright (c) 2006 G. V. R. Babu and K. N. V. V. Vara Prasad. This is an open access article distributed under the Creative Commons Attribution License, which permits unrestricted use, distribution, and reproduction in any medium, provided the original work is properly cited.

\section{Introduction}

By approximation of fixed points of certain classes of operators which satisfy weak contractive-type conditions that do not guarantee the convergence of Picard iteration [2, Example 2.1, page 76], certain mean value fixed point iterations, namely, Krasnoselskij, Mann, and Ishikawa iteration methods are useful to approximate fixed points. For more details on these iterations and further literature, see Berinde [3].

When, for a certain class of mappings, two or more fixed point iteration procedures can be used to approximate their fixed points, it is of theoretical and practical importance to compare the rate of convergence of these iterations, and to find out, if possible, which one of them converges faster. Recent works in this direction are $[1,4,5]$.

Verma [9] approximated fixed points of Lipschitzian and generalized pseudocontractive operators in Hilbert spaces by both Krasnoselskij and Mann iteration, and Berinde [4] established that, for any Mann iteration, there is a Krasnoselskij iteration which converges faster to the fixed point of such an operator.

Chidume and Osilike [7] approximated fixed points of Lipschitzian strongly pseudocontractive maps in Banach spaces, using both Mann and Ishikawa iterations. 
Now, the interest of this paper is to compare the fastness of the convergence to the fixed point among the Krasnoselskij, Mann, and Ishikawa iterations for the class of Lipschitz, strongly pseudocontractive operators in arbitrary real Banach spaces.

\section{Preliminaries and known results}

Suppose that $E$ is a real Banach space with dual $E^{*}$, we denote by $J$, the normalized duality map from $E$ to $2^{E^{*}}$ defined by

$$
J(x)=\left\{f^{*} \in E^{*}:\left\langle x, f^{*}\right\rangle=\|x\|^{2}=\left\|f^{*}\right\|^{2}\right\},
$$

where $\langle\cdot, \cdot\rangle$ denotes the generalized duality pairing.

A mapping $T$ with domain $D(T)$ and range $R(T)$ in $E$ is called Lipschitz, if there exists $L>0$ such that for each $x, y \in D(T)$,

$$
\|T x-T y\| \leq L\|x-y\| .
$$

A mapping $T$ with domain $D(T)$ and range $R(T)$ in $E$ is called strongly pseudocontractive if and only if for any $x, y \in D(T)$, there exists $t>1$ such that

$$
\|x-y\| \leq\|(1+r)(x-y)-r t(T x-T y)\|
$$

for any $r>0$. If $t=1$ in (2.3), then $T$ is called pseudocontractive.

It follows from [8, Lemma 1.1] that $T$ is strongly pseudocontractive if and only if the following condition holds: there exists $j(x-y) \in J(x-y)$ such that

$$
\langle(I-T)(x)-(I-T)(y), j(x-y)\rangle \geq k\|x-y\|^{2}
$$

for each $x, y$ in $E$, where $k=(t-1) / t \in(0,1)$.

Again by using [8, Lemma 1.1] and inequality (2.4) (Bogin [6]) it follows that $T$ is strongly pseudocontractive if and only if the following inequality holds:

$$
\|x-y\| \leq\|x-y+s[(I-T-k I)(x)-(I-T-k I)(y)]\|
$$

for all $x, y \in D(T)$ and $s>0$.

Notation 2.1. Throughout this paper, $E$ denotes a real Banach space, $K$ a closed convex (not necessarily bounded) subset of $E$, and $\operatorname{LS}(K)$ the class of all Lipschitz, strongly pseudocontractive maps on $K$. For any $T \in \mathrm{LS}(K)$, we assume that the Lipschitz constant $L \geq 1$ and pseudocontractive constant $k \in(0,1)$.

Let $x_{0} \in E$ be arbitrary.

(i) For any $\lambda \in(0,1)$, the sequence $\left\{x_{n}\right\}_{n=0}^{\infty} \subseteq E$ defined by

$$
x_{n+1}=T_{\lambda} x_{n}=(1-\lambda) x_{n}+\lambda T x_{n}, \quad n=0,1,2, \ldots,
$$

is called the Krasnoselskij iteration. We denote it by $K\left(x_{0}, \lambda, T\right)$.

(ii) The sequence $\left\{x_{n}\right\}_{n=0}^{\infty} \subseteq E$ defined by

$$
x_{n+1}=\left(1-\alpha_{n}\right) x_{n}+\alpha_{n} T x_{n}, \quad n=0,1,2, \ldots,
$$


where $\left\{\alpha_{n}\right\}_{n=0}^{\infty}$ is a real sequence satisfying $0 \leq \alpha_{n}<1, n=0,1,2, \ldots$, is called the Mann iteration, and is denoted by $M\left(x_{0}, \alpha_{n}, T\right)$.

(iii) The sequence $\left\{x_{n}\right\}_{n=0}^{\infty} \subseteq E$ defined by

$$
\begin{aligned}
x_{n+1} & =\left(1-\alpha_{n}\right) x_{n}+\alpha_{n} T y_{n}, \quad n=0,1,2, \ldots, \\
y_{n} & =\left(1-\beta_{n}\right) x_{n}+\beta_{n} T x_{n}, \quad n=0,1,2, \ldots,
\end{aligned}
$$

where $\left\{\alpha_{n}\right\}_{n=0}^{\infty},\left\{\beta_{n}\right\}_{n=0}^{\infty}$ are sequences of reals satisfying $0 \leq \alpha_{n}, \beta_{n}<1$, is called the Ishikawa iteration, and is denote by $I\left(x_{0}, \alpha_{n}, \beta_{n}, T\right)$.

Chidume and Osilike [7] established the strong convergence of Mann and Ishikawa iterations to the fixed point of $T \in \mathrm{LS}(K)$. Now, the following question arises: for a member T of LS $(K)$ which one of the following, namely, Krasnoselskij, Mann, and Ishikawa iterations converges faster to the fixed point of $T$ ?

To answer this question, we use the following definitions introduced by Berinde [5].

Definition $2.2[5]$. Let $\left\{a_{n}\right\}_{n=0}^{\infty}$ and $\left\{b_{n}\right\}_{n=0}^{\infty}$ be two sequences of real numbers that converge to $a$ and $b$, respectively. Assume that there exists a real number $l$ such that

$$
\lim _{n \rightarrow \infty} \frac{\left|a_{n}-a\right|}{\left|b_{n}-b\right|}=l .
$$

(i) If $l=0$, then $\left\{a_{n}\right\}_{n=0}^{\infty}$ is said to converge faster to $a$ than $\left\{b_{n}\right\}_{n=0}^{\infty}$ to $b$.

(ii) If $0<l<\infty$, then $\left\{a_{n}\right\}_{n=0}^{\infty}$ and $\left\{b_{n}\right\}_{n=0}^{\infty}$ are said to have the same rate of convergence.

Definition 2.3 [5]. Suppose that for two fixed point iteration procedures $\left\{u_{n}\right\}_{n=0}^{\infty}$ and $\left\{v_{n}\right\}_{n=0}^{\infty}$ both converging to the same fixed point $p$ (say) with error estimates

$$
\begin{aligned}
& \left\|u_{n}-p\right\| \leq a_{n}, \quad n=0,1,2, \ldots, \\
& \left\|v_{n}-p\right\| \leq b_{n}, \quad n=0,1,2, \ldots
\end{aligned}
$$

where $\left\{a_{n}\right\}_{n=0}^{\infty}$ and $\left\{b_{n}\right\}_{n=0}^{\infty}$ are two sequences of positive numbers converging to zero. If $\left\{a_{n}\right\}_{n=0}^{\infty}$ converges faster than $\left\{b_{n}\right\}_{n=0}^{\infty}$, then $\left\{u_{n}\right\}_{n=0}^{\infty}$ is said to converge faster than $\left\{v_{n}\right\}_{n=0}^{\infty}$ to $p$.

For more details on definitions, we refer, Berinde [4].

\section{Results on the comparison of fastness of the convergence}

Theorem 3.1. If $T \in \mathrm{LS}(K)$, then the following hold:

(a) for any $x_{0} \in K$ and $\epsilon \in(0, k / 2 M] \cap(0,1)$, the Krasnoselskij iteration $\left\{x_{n}\right\}_{n=0}^{\infty}$ defined by $K\left(x_{0}, \epsilon, T\right)$ converges strongly to the fixed point $x^{*}$ of $T$, where $M=1+(2-$ $k+L)(L+1)$

(b) for any $x_{0} \in K$, the Mann iteration $\left\{x_{n}\right\}_{n=0}^{\infty}$ defined by $M\left(x_{0}, \alpha_{n}, T\right)$ with $\left\{\alpha_{n}\right\}_{n=0}^{\infty} \subset$ $[0,1)$ satisfying (i) $\lim _{n \rightarrow \infty} \alpha_{n}=0$ and (ii) $\sum_{n=0}^{\infty} \alpha_{n}=\infty$ converges strongly to the fixed point $x^{*}$ of $T$; 
4 Comparison of fastness of the convergence

(c) for any $x_{0} \in K$ and for any Mann iteration $\left\{x_{n}\right\}_{n=0}^{\infty}$ defined by $M\left(x_{0}, \alpha_{n}, T\right)$ with $\left\{\alpha_{n}\right\}_{n=0}^{\infty} \subset[0,1)$ satisfying (i) and (ii) of $(b)$, converging to the fixed point $x^{*}$ of $T$, there is an $\epsilon_{0} \in(0,1)$ such that the Krasnoselskij iteration $K\left(x_{0}, \epsilon_{0}, T\right)$ converges faster to the fixed point $x^{*}$ of T. Moreover, $x^{*}$ is unique.

Proof. From [7, Corollary 1], (b) follows. In order to establish (c), we need the following estimates, through which (a) follows.

Using Mann iteration $M\left(x_{0}, \alpha_{n}, T\right)$, from (2.7), we have

$$
\begin{aligned}
x_{n}= & x_{n+1}+\alpha_{n} x_{n}-\alpha_{n} T x_{n}=\left(1+\alpha_{n}\right) x_{n+1}+\alpha_{n}(I-T-k I) x_{n+1} \\
& -(2-k) \alpha_{n} x_{n+1}+\alpha_{n} x_{n}+\alpha_{n}\left(T x_{n+1}-T x_{n}\right)
\end{aligned}
$$

so that

$$
\begin{aligned}
x_{n}-x^{*}= & \left(1+\alpha_{n}\right)\left(x_{n+1}-x^{*}\right)+\alpha_{n}(I-T-k I)\left(x_{n+1}-x^{*}\right) \\
& -(1-k) \alpha_{n}\left(x_{n}-x^{*}\right)+(2-k) \alpha_{n}^{2}\left(x_{n}-T x_{n}\right)+\alpha_{n}\left(T x_{n+1}-T x_{n}\right) .
\end{aligned}
$$

Thus from (2.5), we get

$$
\begin{aligned}
\left\|x_{n}-x^{*}\right\| \geq & \left(1+\alpha_{n}\right)|| x_{n+1}-x^{*}\left\|-(1-k) \alpha_{n}\right\| x_{n}-x^{*} \| \\
& -(2-k) \alpha_{n}^{2}\left\|x_{n}-T x_{n}\right\|-\alpha_{n}\left\|T x_{n+1}-T x_{n}\right\| .
\end{aligned}
$$

Thus

$$
\begin{aligned}
& \left(1+\alpha_{n}\right)\left\|x_{n+1}-x^{*}\right\| \\
& \quad \leq\left[1+(1-k) \alpha_{n}\right]\left\|x_{n}-x^{*}\right\|+(2-k) \alpha_{n}^{2}\left\|x_{n}-T x_{n}\right\|+\alpha_{n}\left\|T x_{n+1}-T x_{n}\right\| .
\end{aligned}
$$

We have

$$
\begin{gathered}
\left\|x_{n}-T x_{n}\right\| \leq\left\|x_{n}-x^{*}\right\|+\left\|x^{*}-T x_{n}\right\| \leq(1+L)\left\|x_{n}-x^{*}\right\|, \\
\left\|T x_{n+1}-T x_{n}\right\| \leq L\left\|x_{n+1}-x_{n}\right\|=L\left\|\left(1-\alpha_{n}\right) x_{n}+\alpha_{n} T x_{n}-x_{n}\right\| \leq L(1+L) \alpha_{n}\left\|x_{n}-x^{*}\right\| .
\end{gathered}
$$

Thus from (3.4), (3.5), we have

$$
\left(1+\alpha_{n}\right)\left\|x_{n+1}-x^{*}\right\| \leq\left[1+(1-k) \alpha_{n}+(2-k) \alpha_{n}^{2}(1+L)+\alpha_{n}^{2} L(1+L)\right] \cdot\left\|x_{n}-x^{*}\right\| .
$$

Now

$$
\begin{aligned}
\left\|x_{n+1}-x^{*}\right\| & \leq\left[\frac{1+(1-k) \alpha_{n}}{1+\alpha_{n}}+(2-k) \alpha_{n}^{2}(1+L)+\alpha_{n}^{2} L(1+L)\right] \cdot\left\|x_{n}-x^{*}\right\| \\
& \leq\left[1-k \alpha_{n}+\alpha_{n}^{2}+\alpha_{n}^{2}(1+L)(2-k+L)\right] \cdot\left\|x_{n}-x^{*}\right\| \\
& =\left[1-k \alpha_{n}+\alpha_{n}^{2}(1+(2-k+L)(1+L))\right] \cdot\left\|x_{n}-x^{*}\right\| .
\end{aligned}
$$


Therefore,

$$
\left\|x_{n+1}-x^{*}\right\| \leq\left[1-k \alpha_{n}+\alpha_{n}^{2} M\right] \cdot\left\|x_{n}-x^{*}\right\|,
$$

where $M=1+(2-k+L)(1+L)$.

On replacing $\alpha_{n}$ by $\epsilon$ in (3.8), we get the following estimate for the Krasnoselskij iteration $K\left(x_{0}, \epsilon, T\right)$ :

$$
\left\|x_{n+1}-x^{*}\right\| \leq\left[1-k \epsilon+\epsilon^{2} M\right] \cdot\left\|x_{n}-x^{*}\right\| .
$$

Here we observe that $1-k \epsilon+\epsilon^{2} M<1$ for any $\epsilon<k / M$. Thus (a) follows.

From the elementary calculus, the function $f$ defined on $[0,1]$ by $f(\epsilon)=[1-k \epsilon+$ $\left.\epsilon^{2} M\right]$ has the minimum value at $\epsilon=\epsilon_{0}$, where $\epsilon_{0}=k / 2 M$.

In particular, for this $\epsilon_{0}>0$, from (3.9), we have the following estimate for the Krasnoselskij iteration:

$$
\left\|x_{n+1}-x^{*}\right\| \leq \theta_{0} \cdot\left\|x_{n}-x^{*}\right\|
$$

where $\theta_{0}=1-\left(k \epsilon_{0} / 2\right)(<1)$.

Thus, inductively it follows that

$$
\left\|x_{n+1}-x^{*}\right\| \leq \theta_{0}^{n} \cdot\left\|x_{1}-x^{*}\right\|
$$

Let $\eta=\min \left\{k / 2 M, k \epsilon_{0}^{2} / 2\right\}$.

Since $\alpha_{n} \rightarrow 0$ as $n \rightarrow \infty$, then there is a positive integer $N_{0}$ such that $\alpha_{n}<\eta$ for all $n \geq N_{0}$.

Then from (3.8), we have

$$
\begin{aligned}
\left\|x_{n+1}-x^{*}\right\| & <\left[1-k \alpha_{n}+\alpha_{n} \eta M\right] \cdot\left\|x_{n}-x^{*}\right\| \quad \forall n \geq N_{0} \\
& \leq\left[1-k \alpha_{n}+\alpha_{n} \frac{k}{2 M} M\right] \cdot\left\|x_{n}-x^{*}\right\| \quad \forall n \geq N_{0} \\
& =\left[1-\frac{k \alpha_{n}}{2}\right] \cdot\left\|x_{n}-x^{*}\right\| \quad \forall n \geq N_{0} .
\end{aligned}
$$

On repeating this process, we get

$$
\left\|x_{n+1}-x^{*}\right\|<\prod_{i=N_{0}}^{n}\left[1-\frac{k \alpha_{i}}{2}\right] \cdot\left\|x_{N_{0}}-x^{*}\right\| \quad \forall n \geq N_{0} .
$$

On comparing the coefficients of the inequalities (3.11) and (3.13) obtained through $K\left(x_{0}, \epsilon_{0}, T\right)$ and $M\left(x_{0}, \alpha_{n}, T\right)$, respectively, we have, for $n \geq N_{0}$,

$$
\frac{\theta_{0}^{n}}{\prod_{i=N_{0}}^{n}\left[1-k \alpha_{i} / 2\right]} \leq \frac{1}{\left(1+k \epsilon_{0} / 2\right)^{n-N_{0}}} \longrightarrow 0 \quad \text { as } n \rightarrow \infty .
$$

Thus by Definition 2.2, the Krasnoselskij iteration converges faster than the Mann iteration to the fixed point $x^{*}$ of $T$. This proves (c). 
Table 3.1

\begin{tabular}{c|cc}
\hline$x_{n}$ & $K\left(x_{0}, \epsilon_{0}, T\right)$ & $K\left(x_{0}, \epsilon, T\right)$ \\
\hline$x_{0}$ & 1.9 & 1.9 \\
\hline$x_{1}$ & 1.875900277 & 1.887950139 \\
$x_{2}$ & 1.852341980 & 1.876035445 \\
$x_{3}$ & 1.829315930 & 1.864254774 \\
$x_{4}$ & 1.806813062 & 1.852606990 \\
$x_{5}$ & 1.784824424 & 1.841090963 \\
$x_{6}$ & 1.763341175 & 1.829705570 \\
$x_{7}$ & 1.742354579 & 1.818449697 \\
$x_{8}$ & 1.721856008 & 1.807322234 \\
$x_{9}$ & 1.701836931 & 1.796322080 \\
$x_{10}$ & 1.682288851 & 1.785448141 \\
\hline
\end{tabular}

Remark 3.2. From (3.10) of Theorem 3.1, it follows that for any $\epsilon \in(0,1)$ with $\epsilon<\epsilon_{0}$, the Krasnoselskij iteration $K\left(x_{0}, \epsilon_{0}, T\right)$ converges faster than $K\left(x_{0}, \epsilon, T\right)$ to the fixed point $x^{*}$ of $T$ for any $x_{0} \in K$. This observation also is numerically shown in Table 3.1.

Theorem 3.3. Let E, $K$, and $T$ be as in Theorem 3.1. Suppose that $\left\{\alpha_{n}\right\}_{n=0}^{\infty}$ and $\left\{\beta_{n}\right\}_{n=0}^{\infty}$ are real sequences in $[0,1)$ such that $\sum_{n=0}^{\infty} \alpha_{n}=\infty$ and $\lim _{n \rightarrow \infty} \alpha_{n}=\lim _{n \rightarrow \infty} \beta_{n}=0$. Then

(a) for any $x_{0} \in K$, the Ishikawa iteration $I\left(x_{0}, \alpha_{n}, \beta_{n}, T\right)$ converges strongly to the fixed point $x^{*}$ of $T$, and

(b) the Mann iteration $M\left(x_{0}, \alpha_{n}, T\right)$ converges faster than the Ishikawa iteration $I\left(x_{0}, \alpha_{n}\right.$, $\left.\beta_{n}, T\right)$ to the fixed point $x^{*}$ of $T$.

Proof. (a) follows from [7, Theorem 1].

We now prove (b). Since $T \in \operatorname{LS}(K)$, from $I\left(x_{0}, \alpha_{n}, \beta_{n}, T\right)$ defined by (2.8), we have

$$
\begin{aligned}
x_{n}= & x_{n+1}+\alpha_{n} x_{n}-\alpha_{n} T y_{n}=\left(1+\alpha_{n}\right) x_{n+1}+\alpha_{n}(I-T-k I) x_{n+1} \\
& -(2-k) \alpha_{n} x_{n+1}+\alpha_{n} x_{n}+\alpha_{n}\left(T x_{n+1}-T y_{n}\right) \\
= & \left(1+\alpha_{n}\right) x_{n+1}+\alpha_{n}(I-T-k I) x_{n+1}-(1-k) \alpha_{n} x_{n} \\
& +(2-k) \alpha_{n}^{2}\left(x_{n}-T y_{n}\right)+\alpha_{n}\left(T x_{n+1}-T y_{n}\right) .
\end{aligned}
$$

Hence

$$
\begin{aligned}
x_{n}-x^{*}= & \left(1+\alpha_{n}\right)\left(x_{n+1}-x^{*}\right)+\alpha_{n}(I-T-k I)\left(x_{n+1}-x^{*}\right) \\
& -(1-k) \alpha_{n}\left(x_{n}-x^{*}\right)+(2-k) \alpha_{n}^{2}\left(x_{n}-T y_{n}\right)+\alpha_{n}\left(T x_{n+1}-T y_{n}\right) .
\end{aligned}
$$

Thus from (2.5), we get

$$
\begin{aligned}
\left\|x_{n}-x^{*}\right\| \geq & \left(1+\alpha_{n}\right)\left\|x_{n+1}-x^{*}\right\|-(1-k) \alpha_{n}\left\|x_{n}-x^{*}\right\| \\
& -(2-k) \alpha_{n}^{2}\left\|x_{n}-T y_{n}\right\|-\alpha_{n}\left\|T x_{n+1}-T y_{n}\right\| .
\end{aligned}
$$


Then

$$
\begin{aligned}
& \left(1+\alpha_{n}\right)\left\|x_{n+1}-x^{*}\right\| \\
& \quad \leq\left[1+(1-k) \alpha_{n}\right]\left\|x_{n}-x^{*}\right\|+(2-k) \alpha_{n}^{2}\left\|x_{n}-T y_{n}\right\|+\alpha_{n}\left\|T x_{n+1}-T y_{n}\right\|
\end{aligned}
$$

We have the following estimates:

$$
\begin{aligned}
\left\|y_{n}-x^{*}\right\| \leq\left(1-\beta_{n}\right)\left\|x_{n}-x^{*}\right\|+\beta_{n}\left\|T x_{n}-x^{*}\right\| \leq\left[1+(L-1) \beta_{n}\right]\left\|x_{n}-x^{*}\right\|, \\
\left\|x_{n}-T y_{n}\right\| \leq\left\|x_{n}-x^{*}\right\|+\left\|x^{*}-T y_{n}\right\| \leq\left\|x_{n}-x^{*}\right\|+L\left\|x^{*}-y_{n}\right\| \\
\leq\left[1+L\left(1+(L-1) \beta_{n}\right)\right]\left\|x_{n}-x^{*}\right\| .
\end{aligned}
$$

Also,

$$
\left\|T x_{n+1}-T x_{n}\right\| \leq L\left\|x_{n+1}-y_{n}\right\| \leq L\left[\left(1-\alpha_{n}\right)\left\|x_{n}-y_{n}\right\|+\alpha_{n}\left\|T y_{n}-y_{n}\right\|\right] .
$$

Now

$$
\begin{gathered}
\left\|T y_{n}-y_{n}\right\| \leq\left\|T y_{n}-x^{*}\right\|+\left\|x^{*}-y_{n}\right\| \leq(1+L)\left\|y_{n}-x^{*}\right\|, \\
\left\|x_{n}-y_{n}\right\|=\beta_{n}\left\|x_{n}-T x_{n}\right\| \leq(1+L) \beta_{n}\left\|x_{n}-x^{*}\right\| .
\end{gathered}
$$

Now on substituting (3.22) in (3.21) and using (3.19), we have

$$
\begin{aligned}
\left\|T x_{n+1}-T y_{n}\right\| & \leq L\left[(1+L)\left(1-\alpha_{n}\right) \beta_{n}+\alpha_{n}(1+L)\left(1+(L-1) \beta_{n}\right)\right]\left\|x_{n}-x^{*}\right\| \\
& =L(1+L)\left[\left(1-\alpha_{n}\right) \beta_{n}+\alpha_{n}\left(1+(L-1) \beta_{n}\right)\right]\left\|x_{n}-x^{*}\right\| .
\end{aligned}
$$

On using (3.20) and (3.23) in (3.18), we get

$$
\begin{aligned}
\left(1+\alpha_{n}\right)\left\|x_{n+1}-x^{*}\right\| \leq[1 & +(1-k) \alpha_{n}+\alpha_{n}^{2}(2-k)\left[1+L\left(1+(L-1) \beta_{n}\right)\right] \\
& \left.+\alpha_{n} L(1+L)\left[\left(1-\alpha_{n}\right) \beta_{n}+\alpha_{n}\left(1+(L-1) \beta_{n}\right)\right]\right]\left\|x_{n}-x^{*}\right\| \\
< & {\left[1+(1-k) \alpha_{n}+\alpha_{n}^{2}(2-k+L)(1+L)+\gamma\left(\alpha_{n}, \beta_{n}, L, k\right)\right]\left\|x_{n}-x^{*}\right\|, }
\end{aligned}
$$

where

$$
\gamma\left(\alpha_{n}, \beta_{n}, L, k\right)=\alpha_{n} \beta_{n} L\left[(2-k)(L-1)+(L+1)\left(1-\alpha_{n}\right)+(1+L)(L-1)\right]\left\|x_{n}-x^{*}\right\| .
$$

Thus

$$
\begin{aligned}
\left\|x_{n+1}-x^{*}\right\| & \leq\left[\frac{1+(1-k) \alpha_{n}}{1+\alpha_{n}}+\alpha_{n}^{2}(2-k+L)(1+L)+\gamma\left(\alpha_{n}, \beta_{n}, L, k\right)\right]\left\|x_{n}-x^{*}\right\| \\
& =\left[1-k \alpha_{n}+\alpha_{n}^{2}+\alpha_{n}^{2}(2-k+L)(1+L)+\gamma\left(\alpha_{n}, \beta_{n}, L, k\right)\right]\left\|x_{n}-x^{*}\right\| \\
& =\left[1-k \alpha_{n}+\alpha_{n}^{2}(1+(2-k+L)(1+L))+\gamma\left(\alpha_{n}, \beta_{n}, L, k\right)\right]\left\|x_{n}-x^{*}\right\| .
\end{aligned}
$$


8 Comparison of fastness of the convergence

Define $M_{1}=(3-k+L)(1+L)$.

Since

$$
\begin{gathered}
1+(2-k+L)(1+L) \leq M_{1}, \\
(2-k)(L-1)+(L+1)\left(1-\alpha_{n}\right)+(1+L)(L-1) \leq M_{1},
\end{gathered}
$$

we have

$$
\gamma\left(\alpha_{n}, \beta_{n}, L, k\right) \leq \alpha_{n} \beta_{n} L M_{1}
$$

Now (3.26) becomes

$$
\begin{aligned}
\left\|x_{n+1}-x^{*}\right\| & \leq\left[1-k \alpha_{n}+\alpha_{n}^{2} M_{1}+\alpha_{n} \beta_{n} L M_{1}\right]\left\|x_{n}-x^{*}\right\| \\
& =\left[1-k \alpha_{n}+\alpha_{n}\left(\alpha_{n}+\beta_{n} L\right) M_{1}\right]\left\|x_{n}-x^{*}\right\| .
\end{aligned}
$$

Since $\alpha_{n} \rightarrow 0$ as $n \rightarrow \infty$, there is a positive integer $N_{0}$ such that

$$
\alpha_{n}<\frac{k \epsilon_{0}}{2 M_{1}} \quad \forall n \geq N_{0},
$$

and since $\beta_{n} \rightarrow 0$ as $n \rightarrow \infty$, there is a positive integer $N_{1}$ such that

$$
\beta_{n}<\frac{k \epsilon_{0}}{2 M_{1} L} \quad \forall n \geq N_{1} .
$$

Write $N=\max \left\{N_{0}, N_{1}\right\}$. Now for any $n \geq N$, (3.29) becomes

$$
\begin{aligned}
\left\|x_{n+1}-x^{*}\right\| & <\left[1-k \alpha_{n}+\alpha_{n}\left(\frac{k \epsilon_{0}}{2 M_{1}}+\frac{k \epsilon_{0}}{2 M_{1} L} L\right) M_{1}\right]\left\|x_{n}-x^{*}\right\| \\
& =\left[1-k \alpha_{n}\left(1-\epsilon_{0}\right)\right]\left\|x_{n}-x^{*}\right\| .
\end{aligned}
$$

On repeating this process, we get

$$
\left\|x_{n+1}-x^{*}\right\|<\left\{\prod_{i=N}^{n}\left[1-k \alpha_{i}\left(1-\epsilon_{0}\right)\right]\right\}\left\|x_{N}-x^{*}\right\| \quad \forall n \geq N,
$$

which is an estimation for the Ishikawa iteration $I\left(x_{0}, \alpha_{n}, \beta_{n}, T\right)$.

On choosing $\beta_{n}=0$ for all $n$, in (3.29), we get the following estimate for Mann iteration $M\left(x_{0}, \alpha_{n}, T\right)$ :

$$
\begin{aligned}
\left\|x_{n+1}-x^{*}\right\| & \leq\left[1-k \alpha_{n}+\alpha_{n}^{2} M_{1}\right]\left\|x_{n}-x^{*}\right\| \\
& <\left[1-k \alpha_{n}+\alpha_{n} M_{1} \frac{k \epsilon_{0}}{2 M_{1}}\right]\left\|x_{n}-x^{*}\right\| \quad \forall n \geq N \\
& =\left[1-k \alpha_{n}\left(1-\frac{\epsilon_{0}}{2}\right)\right]\left\|x_{n}-x^{*}\right\| \quad \forall n \geq N .
\end{aligned}
$$


On repeating process, we get

$$
\left\|x_{n+1}-x^{*}\right\|<\left\{\prod_{i=N}^{n}\left[1-k \alpha_{i}\left(1-\frac{\epsilon_{0}}{2}\right)\right]\right\}\left\|x_{N}-x^{*}\right\| .
$$

On comparing the coefficients of the inequalities (3.33) and (3.35), we get that for any $n \geq N$,

$$
\frac{\prod_{i=N}^{n}\left[1-k \alpha_{i}\left(1-\epsilon_{0} / 2\right)\right]}{\prod_{i=N}^{n}\left[1-k \alpha_{i}\left(1-\epsilon_{0}\right)\right]} \leq \prod_{i=N}^{n}\left[1-k \alpha_{i} \frac{\epsilon_{0}}{2}\right] .
$$

Since $\sum_{n=0}^{\infty} \alpha_{n}=\infty$, we have $\lim _{n \rightarrow \infty} \prod_{i=N}^{n}\left[1-k \alpha_{i}\left(\epsilon_{0} / 2\right)\right]=0$. Thus the Mann iteration $M\left(x_{0}, \alpha_{n}, T\right)$ converges faster than the Ishikawa iteration $I\left(x_{0}, \alpha_{n}, \beta_{n}, T\right)$ to the fixed point of $T$.

Remark 3.4. Under the assumptions of Theorem 3.1, it follows that for any Mann iteration $M\left(x_{0}, \alpha_{n}, T\right)$ there is a Krasnoselskij iteration $K\left(x_{0}, \epsilon_{0}, T\right)$ converges faster to the fixed point of $T$; and from Theorem 3.3 it follows that the Mann iteration $M\left(x_{0}, \alpha_{n}, T\right)$ converges faster than the Ishikawa iteration $I\left(x_{0}, \alpha_{n}, \beta_{n}, T\right)$ to the fixed point of $T$. Hence we conclude that the Krasnoselskij iteration converges faster than both the Mann and Ishikawa iterations to the fixed point of $T \in \mathrm{LS}(K)$.

\section{Numerical examples}

The following examples show the fastness of the movement of the first 10 iterates towards the fixed point.

Example 4.1 [4]. Let $X=[1 / 2,2]$ and $T: X \rightarrow X$ given by $T x=1 / x$ for all $x \in X$. Then $T$ is Lipschitz with Lipschitzian constant $L=4$; and is strongly pseudocontractive with any positive constant $k \in(0,1)$.

We note that Picard iteration does not converge for any $x_{0} \neq 1$ in $X$.

From Theorems 3.1 and 3.3, we have the following.

(i) The Krasnoselskij iteration $K\left(x_{0}, \epsilon_{0}, T\right)$ converges to the fixed point $x^{*}=1$, where $\epsilon_{0}=k / 2 M$, in which $k \in(0,1)$ and $M=31-5 k$. Choosing $k=62 / 67$, we have $\epsilon_{0}=1 / 57$. For this $\epsilon_{0}$, the Krasnoselskij iteration $K\left(x_{0}, \epsilon_{0}, T\right)$ is given by

$$
x_{n+1}=\frac{1}{57}\left(56 x_{n}+x_{n}^{-1}\right), \quad n=0,1,2, \ldots,
$$

which converges to the fixed point $x^{*}=1$.

(ii) Also with $\alpha_{n}=1 /(n+58), n=0,1,2, \ldots$, the corresponding Mann iteration $M\left(x_{0}\right.$, $\left.\alpha_{n}, T\right)$ is given by

$$
x_{n+1}=\frac{1}{n+58}\left[(n+57) x_{n}+x_{n}^{-1}\right], \quad n=0,1,2, \ldots
$$

which converges to $x^{*}=1$. 
Table 4.1

\begin{tabular}{c|ccc}
\hline$x_{n}$ & $K\left(x_{0}, \epsilon_{0}, T\right)$ & $M\left(x_{0}, \alpha_{n}, T\right)$ & $I\left(x_{0}, \alpha_{n}, \alpha_{n}, T\right)$ \\
\hline$x_{0}$ & 1.9 & 1.9 & 1.9 \\
\hline$x_{1}$ & 1.875900277 & 1.876315789 & 1.876430333 \\
$x_{2}$ & 1.852341980 & 1.853547036 & 1.853770048 \\
$x_{3}$ & 1.829315930 & 1.831646354 & 1.831972078 \\
$x_{4}$ & 1.806813062 & 1.810569477 & 1.810992457 \\
$x_{5}$ & 1.784824424 & 1.790275008 & 1.790790067 \\
$x_{6}$ & 1.763341175 & 1.770724189 & 1.771324237 \\
$x_{7}$ & 1.742354579 & 1.751880697 & 1.752563291 \\
$x_{8}$ & 1.721856008 & 1.733710457 & 1.734471166 \\
$x_{9}$ & 1.701836931 & 1.716181474 & 1.717016088 \\
$x_{10}$ & 1.682288851 & 1.699263676 & 1.700168192 \\
\hline
\end{tabular}

(iii) The Ishikawa iteration $I\left(x_{0}, \alpha_{n}, \beta_{n}, T\right)$ converges to $x^{*}=1$ with $\alpha_{n}=\beta_{n}=1 /(n+58)$, $n \geq 0$. In this case, the sequence $I\left(x_{n}, \alpha_{n}, \alpha_{n}, T\right)$ is given by

$$
x_{n+1}=\frac{n+57}{n+58} x_{n}+\frac{x_{n}}{(n+57) x_{n}^{2}+1}, \quad n=0,1,2, \ldots
$$

(iv) The comparison of the fastness of first 10 iterates of the Krasnoselskij, Mann, and Ishikawa iterations to the fixed point $x^{*}=1$ is given in Table 4.1 with $x_{0}=1.9$, and $\alpha_{n}=1 /(n+58)$ with $\epsilon_{0}=1 / 57$.

From Table 4.1, we observe that the Krasnoselskij iteration moves faster towards the fixed point $x^{*}=1$.

(v) Table 3.1 shows the comparison of first 10 iterates of Krasnoselskij iterations $K\left(x_{0}\right.$, $\epsilon, T)$ and $K\left(x_{0}, \epsilon_{0}, T\right)$, where $\epsilon=1 / 114, \epsilon_{0}=1 / 57$, and $x_{0}=1.9$. Here we observe that $K\left(x_{0}, \epsilon_{0}, T\right)$ moves faster than $K\left(x_{0}, \epsilon, T\right)$ to the fixed point $x^{*}=1$ of $T$ (see Remark 3.2).

Example 4.2. Let $X=[0,1]$ and $T: X \rightarrow X$ given by $T x=1-x^{2}$ for all $x \in X$. Then $T$ is Lipschitz, with Lipschitzian constant $L=2$, and is strongly pseudocontractive with any positive constant $k \in(0,1)$.

(i) From Theorem 3.1, the Krasnoselskij iteration $K\left(x_{0}, \epsilon_{0}, T\right)$ converges to $x^{*}=$ $(\sqrt{5}-1) / 2$, where $\epsilon_{0}=k / 2 M, k \in(0,1)$, and $M=13-3 k$.

Let $x_{0}=0.9$. Now for $k=26 / 27$, we have $\epsilon_{0}=1 / 21$; thus the Krasnoselskij iteration $K\left(x_{0}, \epsilon_{0}, T\right)$ is given by

$$
x_{n+1}=\frac{1}{21}\left[1+x_{n}\left(20-x_{n}\right)\right], \quad n=0,1,2, \ldots
$$

(ii) The Mann iteration $M\left(x_{0}, \alpha_{n}, T\right)$ converges to $x^{*}=(\sqrt{5}-1) / 2$, where $\alpha_{n}=1 /(n+$ 22), $n=0,1,2, \ldots$, and the Mann iteration $M\left(x_{0}, \alpha_{n}, T\right)$ is given by

$$
x_{n+1}=\frac{1}{n+22}\left[1+x_{n}\left(n+21-x_{n}\right)\right], \quad n=0,1,2, \ldots
$$


Table 4.2

\begin{tabular}{c|ccc}
\hline$x_{n}$ & $K\left(x_{0}, \epsilon_{0}, T\right)$ & $M\left(x_{0}, \alpha_{n}, T\right)$ & $I\left(x_{0}, \alpha_{n}, \alpha_{n}, T\right)$ \\
\hline$x_{0}$ & 0.9 & 0.9 & 0.9 \\
\hline$x_{1}$ & 0.866190476 & 0.867727272 & 0.870320426 \\
$x_{2}$ & 0.836834456 & 0.840741276 & 0.848058999 \\
$x_{3}$ & 0.811257010 & 0.817925143 & 0.826070151 \\
$x_{4}$ & 0.788904869 & 0.798448075 & 0.807059203 \\
$x_{5}$ & 0.769320309 & 0.781680098 & 0.790510886 \\
$x_{6}$ & 0.752121544 & 0.767135510 & 0.776017193 \\
$x_{7}$ & 0.736987546 & 0.754434352 & 0.763251561 \\
$x_{8}$ & 0.723647632 & 0.743275540 & 0.751950035 \\
$x_{9}$ & 0.711870797 & 0.733417737 & 0.741897348 \\
$x_{10}$ & 0.701459805 & 0.724665501 & 0.732916484 \\
\hline
\end{tabular}

(iii) The Ishikawa iteration $I\left(x_{0}, \alpha_{n}, \beta_{n}, T\right)$ converges to the fixed point $x^{*}=(\sqrt{5}-1) / 2$ by Theorem 3.3 with $\alpha_{n}=\beta_{n}=1 /(n+22), n=0,1,2, \ldots$. The Ishikawa iteration $I\left(x_{0}, \alpha_{n}, \alpha_{n}, T\right)$ is given by

$$
x_{n+1}=\left(\frac{n+21}{n+22}\right) x_{n}+\frac{1}{n+22}\left[1-\left(\frac{1}{n+22}\left(1+x_{n}\left(n+21-x_{n}\right)\right)\right)^{2}\right], \quad n=0,1,2, \ldots
$$

(iv) Comparison of Krasnoselskij, Mann, and Ishikawa iterations is given for first 10 iterates in Table 4.2 for $x_{0}=0.9$, and $\alpha_{n}=1 /(n+22)$ with $\epsilon_{0}=1 / 21$.

\section{Acknowledgments}

The authors thank the referees for their valuable suggestions which improved the presentation of the paper. The authors express their heart felt thanks to Prof. Vasile Berinde for providing the reprints of his numerous valuable papers. This work is partially supported by UGC Major Research Project Grant no. F. 8-8/2003 (SR). The first author thanks the University Grants Commission, India, for the financial support.

\section{References}

[1] G. V. R. Babu and K. N. V. V. Vara Prasad, Mann iteration converges faster than Ishikawa iteration for the class of Zamfirescu operators, Fixed Point Theory and Applications 2006 (2006), Article ID 49615, 6 pages.

[2] V. Berinde, Approximating fixed points of Lipschitzian generalized pseudo-contractions, Mathematics \& Mathematics Education (Bethlehem, 2000), World Scientific, New Jersey, 2002, pp. $73-81$.

[3] _ Iterative Approximation of Fixed Points, Editura Efemeride, Baia Mare, 2002.

[4] __ Comparing Krasnoselskij and Mann iterative methods for Lipschitzian generalized pseudocontractions, Proceedings of International Conference on Fixed Point Theory and Applications. Valencia(Spain), 2003, Yokohama Publishers, Yokohama, 2004, pp. 15-26. 


\section{Comparison of fastness of the convergence}

[5] __ Picard iteration converges faster than Mann iteration for a class of quasi-contractive operators, Fixed Point Theory and Applications 2004 (2004), no. 2, 97-105.

[6] J. Bogin, On strict pseudo-contractions and fixed point theorems, Technion preprint, series no: MT-219, Haifa, Israel, 1974.

[7] C. E. Chidume and M. O. Osilike, Nonlinear accretive and pseudo-contractive operator equations in Banach spaces, Nonlinear Analysis. Theory, Methods \& Applications 31 (1998), no. 7, 779789.

[8] T. Kato, Nonlinear semigroups and evolution equations, Journal of the Mathematical Society of Japan 19 (1967), 508-520.

[9] R. U. Verma, A fixed-point theorem involving Lipschitzian generalised pseudo-contractions, Proceedings of the Royal Irish Academy. Section A 97 (1997), no. 1, 83-86.

G. V. R. Babu: Department of Mathematics, Andhra University, Visakhapatnam 530 003, India E-mail address: gvr_babu@hotmail.com

K. N. V. V. Vara Prasad: Department of Mathematics, Dr. L. Bullayya College, Visakhapatnam 530 013, India

E-mail address: knvp71@yahoo.co.in 